\title{
FxkR Provides the Missing Link in the fixL-fixK Signal Transduction Cascade in Rhizobium etli CFN42
}

\author{
David Zamorano-Sánchez, ${ }^{1}$ Alma Reyes-González,, ${ }^{1}$ Nicolás Gómez-Hernández,, ${ }^{1,2}$ Patricia Rivera, ${ }^{1}$ \\ Dimitris Georgellis, ${ }^{3}$ and Lourdes Girard ${ }^{1}$ \\ ${ }^{1}$ Programa de Genómica Funcional de Procariotes, Centro de Ciencias Genómicas, Universidad Nacional Autónoma de \\ México, Avenida Universidad 1001, Cuernavaca, Morelos, 62209, México; ${ }^{2}$ National Center for Soybean Biotechnology, \\ Division of Plant Sciences, University of Missouri, Columbia 65211, U.S.A.; ${ }^{3}$ Departamento de Genética Molecular, Instituto \\ de Fisiología Celular, Universidad Nacional Autónoma de México, Ciudad Universitaria, México D.F. 04510, México
}

Submitted 28 May 2012. Accepted 8 July 2012.

Transcriptional control of the fixK gene in Rhizobium etli and $R$. leguminosarum bv. viciae is governed by a two-component signal transduction system that diverts from the conventional FixL-FixJ cascade that occurs in model rhizobia. Although a fix $L$ gene, encoding a hybrid histidine kinase (hFixL), is present in $R$. etli, no fix J, the cognate response regulator, has been identified. In this work, we present evidence that the pRet42f-located open reading frame RHE PF00530 $(f x k R)$ encodes a novel response regulator indispensable for fixKf activation under microaerobic growth. Moreover, results from complementation assays demonstrate that the activation of fix $f$ expression requires the presence of both hFixL and FxkR, and that the $f x k R$ ortho$\log$ from $R$. leguminosarum bv. viciae is able to substitute for FxkR transcriptional control in $R$. etli. In addition, in these two organisms, hFixL- and FxkR-related proteins were identified in other bacteria, located in close proximity to a fixK-related gene. Using reporter fusions, site-directed mutagenesis, and electrophoretic mobility shift assays, we identified the FxkR binding site upstream from the transcriptional start site of fix $K$ f. Similar to our previous observations for fixL and fixKf mutants, a null mutation in fxkR does not affect the symbiotic effectiveness of the strain. Thus, our findings reveal that FxkR is the longstanding missing key regulator that allows the transduction of the microaerobic signal for the activation of the FixKf regulon.

Nitrogen fixation by soil bacteria in association with leguminous plants requires the concerted expression of genes by both partners. Many regulatory circuits operate inside the nodule to carry out bacterial differentiation into nitrogen-fixing bacteroids and for the nitrogen fixation process per se. Research on the regulation of biological nitrogen fixation has gained interest during the last four decades. Forward and reverse genetic approaches have paved the way to describe key regulators and

Corresponding author: L. Girard; E-mail: girard@ccg.unam.mx

Nucleotide sequence of the Rhizobium leguminosarum bv. viciae VF39 $f x k R$ gene is available in the EMBL Nucleotide Sequence Database under accession number FR854234.

* The $\boldsymbol{e}$-Xtra logo stands for "electronic extra" and indicates that three supplementary figures and three supplementary tables are published online. their involvement in different regulatory cascades in rhizobia. Regulatory pathways of genes involved in nitrogen fixation are integrated in two different networks in a species-specific manner (Fischer 1994; Kaminski et al. 1998). Expression of nif and fix genes is under the control of several regulatory proteins, where low oxygen concentration operates as the most important regulatory signal. For most rhizobia, two regulatory cascades have been described. One includes the NifA and RpoN regulators, while FixL, FixJ, and FixK compose the other regulatory cascade, specific for symbiotic diazotrophs (Dixon and Khan 2004; Fischer 1994).

The regulatory control of nitrogen fixation genes involving FixLJ and FixK possess, as a common function, the regulation of fixNOQP and fixGHIS genes. FixL and FixJ are members of the two-component regulatory systems and FixK is a transcriptional regulator that belongs to the Crp/Fnr protein family. fixK and the cytochrome oxidase complex encoded by fixNOQP have been shown to participate in not only nitrogen fixation but also important physiological pathways such as photosynthesis, carbon, nitrogen, or iron metabolism, and hypoxia or anoxia responses in $\alpha$-proteobacteria such as Rhodopseudomonas palustris and Caulobacter crescentus (Bose and Newman 2011; Britos et al. 2011; Crosson et al. 2005; Rey and Harwood 2010). The fixL and fixJ genes were described as a two-gene operon crucial for the activation of diverse nitrogen fixation genes, including fixK and nifA, under symbiotic and free-living microaerobic conditions in Sinorhizobium meliloti (David et al. 1988). At low oxygen concentration, FixL catalyzes its own phosphorylation with ATP. FixL-P then transfers the phosphate to the transcription factor FixJ in an oxygen-independent reaction (Gilles-Gonzalez and Gonzalez 1993). FixJ-P acts, in turn, as a positive regulator of the fix $K$ gene necessary for the fixLJ regulatory function (Batut et al. 1989). To date, the presence of homologues of the $S$. meliloti fix $L$, fixJ, and fixK genes is a common feature among the different nitrogen fixers. However, the connectivity among the different cascades, the specific regulatory role of each element, and their target genes may vary (Anthamatten et al. 1991, 1992; Bauer et al. 1998; Fischer 1994; Kaminski et al. 1998).

Rhizobium etli CFN42 fixes nitrogen in association with bean plants and its genome contains a chromosome and six large plasmids (pRet42a to pRet42f) whose sizes range from 184.4 to $642.5 \mathrm{~kb}$ (González et al. 2006). Previously, we described that $R$. etli CFN42 employs a complex regulatory circuit for fix genes that integrates several novel characteristics 
when compared with what are considered the conventional systems, S. meliloti and Bradyrhizobium japonicum. Interestingly, important regulatory elements, such as fixL and fixK that are found on the symbiotic plasmid of $S$. meliloti, appear on a different replicon in $R$. etli. fix $L$ encodes an orphan histidine kinase located in a pRet42f region containing copies of the fixK, fixNOQP, and fixGHIS genes. fixNOQP reiterations are controlled by a novel fixL-fixKf cascade, where a fixJ gene is absent and the response regulator that activates transcription of fixKf remains to be described (Girard et al. 1996, 2000; González et al. 2003, 2006). The FixL protein of $R$. etli CFN42 is a hybrid histidine kinase (hHK) with no predicted transmembrane segments and, herein, we will differentiate the typical FixL from the hybrid one by denoting the latter group as hFixL. Also, no response regulator that could be proposed as the cognate response regulator is encoded in the vicinity of hfixL.

The genome of $R$. etli CFN42 codes six fnr-related genes: fixKf, fnrNch, fnrNd, nnrR, stoRd, and stoRf (González et al. 2006). The expression of these genes in $R$. etli depends on the FixKf regulator in microaerobic free-living conditions (Girard et al. 2000; Granados-Baeza et al. 2007; López et al. 2001). However, the complete regulatory pathway that controls expression of the FixK regulatory targets where an hfixL gene is involved has not yet been reported. The response regulator that activates fixK in coordination with hFixL might be a central distributor of the hypoxic response not only for nitrogen fixation in rhizobia but also for other important microaerobic traits in $\alpha$-proteobacteria. The lack of sequences with a high percentage of identity to FixJ and the absence of the two reported consensus sequences for binding of FixJ (Ferrières and Kahn 2002) in the regulatory region of fix Kf are suggestive of a novel two-component system operating in $R$. etli.

A similar distribution of fix genes is shared by R. leguminosarum bv. viciae VF39 (Patschkowski et al. 1996). The regulatory circuit of fix genes is also composed of an orphan hFixL histidine kinase and two fnr homologues ( $f i x K$ and $f n r N$ ). Both $f n r$ homologues seem to be necessary for optimal nitrogen fixation, although $f n r N$ appears to be the central regulator of the fixNOQP reiterations (Schlüter et al. 1997).

hHK that share features of hFixL were found in different genomes, and all these proteins have unorthodox $\mathrm{H}$ boxes when compared with the classic HK domain in other FixL histidine kinases (Boesten and Priefer 2004). The His ${ }^{200}$ and $\mathrm{Arg}^{220}$ residues in B. japonicum FixL that are involved in heme coordination and oxygen binding (Dunham et al. 2003; Gong et al. 1998) are conserved in most described FixL and hFixL proteins (Boesten and Priefer 2004). It is important to remark that the only two models where an $h f i x L$ has been studied lack a fixJ gene, and the cognate response regulator of hFixL is waiting to be discovered (González et al. 2006; Young et al. 2006). Bacteria that encode hFixL-related proteins might share this novel response regulator.

This work uncovers a novel central response regulator of a widely studied regulatory circuit in $R$. etli CFN42 that provides the missing link in the fix $L$-fixK regulatory network for fix gene expression. Also, our findings permit us to propose that similar circuits could operate in different genera of rhizobia and probably also in non-nitrogen-fixing $\alpha$-proteobacteria.

\section{RESULTS}

Probing for the genetic elements needed for fixKf activation in $R$. etli.

Total sequence analysis revealed that no sequences with a high percentage of identity to FixJ are present in $R$. etli CFN42. Nevertheless, because plasmids pRet42d and pRet42f contain most of the fix genes (Girard et al. 1996, 2000; González et al. 2003, 2006), the participation of these two megaplasmids in fixKf microaerobic expression was evaluated. Plasmid pLG1 containing a fixKf::uidA transcriptional fusion (Girard et al. 2000) was introduced in different plasmid-containing derivatives of $R$. etli CFN42. The results show that the microaerobic expression of fixKf was observed in all tested genetic backgrounds, with the exception of strains lacking pRet42f (CFNX186, a CE3 derivative cured of Ret42f or CFNX218 carrying only a partially deleted pRet42e that retains the fragment that is indispensable for cell viability) (Fig. 1) (Brom et al. 1992). Expression of a gene expressed constitutively ( $\arg C)$ is not affected by the loss of this replicon (data not shown). Considering that the presence of hFixL is indispensable for fixKf microaerobic expression (Girard et al. 2000) and that this gene is located on pRet42f, loss of fixKf expression in strains lacking this plasmid (CFNX186 and CFNX218) was expected (Girard et al. 2000). Consequently we cannot affirm that the response regulator responsible for fix $K f$ activation is encoded on this plasmid. Nevertheless, microaerobic expression of fixKf in a derivative carrying only pRet $42 \mathrm{f}$ and the deleted pRet42e (CFNX218/pRet42f) proceeded at similar levels when compared with the wild-type strain (Fig. 1). Therefore, it can be concluded that the activator of fix Kf expression could be located in pRet42f, the partially deleted pRet42e, or the chromosome. Preservation of fix $K \mathrm{f}$ expression in a CE3 derivative that was cured of pRet42d (strain CFNX89) (Brom et al. 1992) revealed that this plasmid was dispensable for the activation of this gene. The subtle induction of expression observed in this strain could be explained by the absence of StoRd, a negative transcriptional regulator in $R$. etli CFN42 (Granados-Baeza et al. 2007).

\section{Identification of FxkR as the cognate response regulator for the hFixL sensor kinase in $\boldsymbol{R}$. etli.}

To identify a response regulator analogous to fixJ, we looked for the presence of response regulators in the $R$. etli CFN42 genome, using the database resource P2CS (Barakat et al. 2011). According to this database, 68 open reading frames (ORF) were predicted as response regulators; of those, 23 are

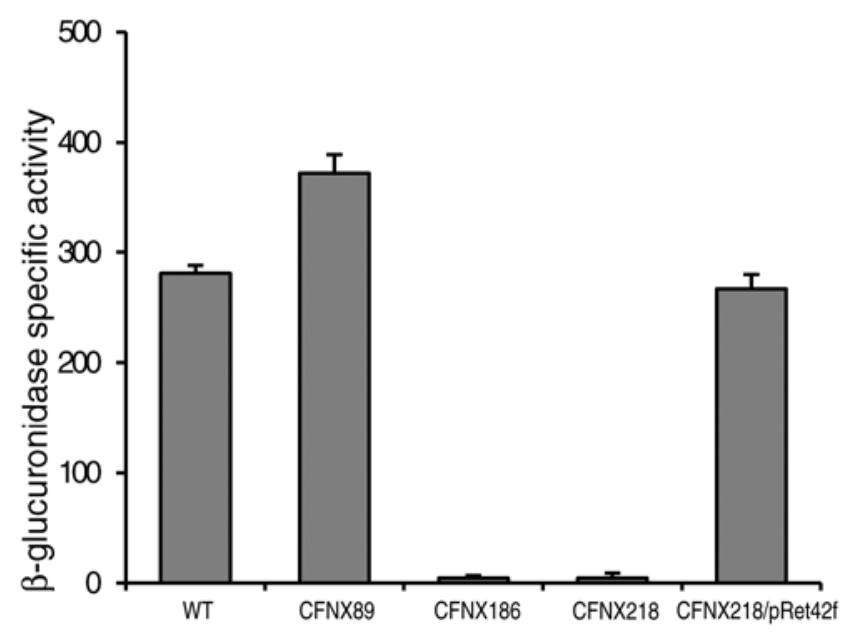

Fig. 1. Participation of Rhizobium etli pRet $42 \mathrm{~d}$ and pRet $42 \mathrm{f}$ plasmids in the regulation of fix $\mathrm{f}$ microaerobic expression. Expression of fix Kf was analyzed under low oxygen concentration $(1 \%)$ in different $R$. etli derivatives: CE3 (wild type), CFNX89 (cured of pRet42d), CFNX186 (cured of Ret42f), CFNX218 (carrying only a partially deleted pRet42e), and CFNX218 containing pRet42f. Cells were microaerobically induced for $10 \mathrm{~h}$. $\beta$-glucuronidase specific activity is expressed as nanomoles of product per minute per milligram of protein. Data are the mean of two replicas from three independent experiments. 
codified in different megaplasmids. In all, 14 response regulators were encoded in pRet $42 \mathrm{f}$ while only 2 were found in the partially deleted pRet $42 \mathrm{e}$. Two proteins from plasmid pRet $42 \mathrm{f}$ (RHE_PF00225 and RHE_PF00548) had significant sequence identity with FixJ from S. meliloti: 40 and $37 \%$, respectively (genetic localization of the putative response regulators in pRet42f is illustrated in Supplementary Figure S1).

Following the rational that pRet $42 \mathrm{f}$ encodes the hFixL sensor and the FixKf regulator, in combination with the fact that pRet42f is able to complement strain CFNX218 for fixKf expression, we hypothesized that the activator of fixKf might also be located in this replicon. By vector-insertion mutagenesis (discussed below), we inactivated the two fixJ-related genes and six additional genes that encode putative response regulators in pRet42f. Genes RHE_PF00530 and RHE_PF00533, belonging to the OmpR/PhoB and NarL/FixJ superfamily, respectively, encode orphan response regulators. Genes RHE_ PF00225, RHE_PF00479, and RHE_PF00548 belong to the NarL/FixJ superfamily and appear to be part of an operon that includes a histidine kinase. Finally, the three additional genes RHE_PF00072, RHE_PF00547, and RHE_PF00224 have no identifiable DNA-binding domain in the SMART database (Letunic et al. 2009), and the first one has an additional nucleotide-binding domain. These genes also appear to be part of a probable operon that includes a histidine kinase (Rhizobase Database).

The microaerobic expression of fixKf was studied by introducing plasmid pLG1 containing a fixKf::uidA transcriptional fusion (pBBMCS53::fixKf) (Girard et al. 2000) in the wild type and in various response regulator mutant backgrounds (SR72/pLG1， SR224/pLG1， SR225/pLG1， SR479/pLG1, SR530/pLG1, SR533/pLG1, SR547/pLG1, and SR548/pLG1). Our results clearly show that the microaerobic expression of fixKf is fully dependent on a functional protein encoded by ORF RHE_PF00530 (Table 1, line 6). No other response regulator mutant, including the FixJ-related proteins, had an effect on the microearobic expression of fixKf. Gene RHE_PF00530 encodes a polypeptide of 241 amino acids and is located 19.5 $\mathrm{kb}$ downstream from the hfixL gene. Here, we designate this gene $f x k R$ (for fixK regulator). It is noteworthy that FxkR is a response regulator that does not belong to the NarL/FixJ superfamily but to the OmpR/PhoB one.

To determine whether the regulatory activity of FxkR depends on an active hFixL, the expression pattern of fix $\mathrm{Kf}$ in the wild-type strain and the fixL::loxSp (CFNX636) and fxkR:: loxSp (CFN-LG1) mutants was monitored, making use of the reporter fusion in pLG1. The microaerobic expression of fix $\mathrm{Kf}$ was completely dependent on the hFixL and FxkR regulators (Table 2, compare lines 1 to 3). Complementation analysis of the $f x k R$ mutant strain with plasmid $\mathrm{p} f x k R$-Ret, containing the wild-type $f x k R$ gene under the control of the lacZ promoter, restored the maximal fixKf microaerobic expression (Table 2, compare lines 1 and 3 with line 5) but failed to restore fix $K \mathrm{f}$ expression to wild-type levels in the hfixL mutant (Table 2, compare lines 1 and 2 with line 4). The higher level of fixKf expression in the complemented $f x k R$ mutant was probably due to multiple copies of the plasmid-borne activator. These results clearly demonstrate that both FxkR and hFixL are required for activation of fix $K \mathrm{f}$ expression.

These results also suggest that dependence for hFixL is not through the transcriptional control of the FxkR regulator. To corroborate this, the promoter region of $f x k R$ was fused with a promoterless $\beta$-glucuronidase (GUS) gene (pARG2). This plasmid was introduced into $R$. etli strains CE3 (wild-type), CFNX636 (fixL::loxSp), and CFN-LG1 (fxkR::loxSp). Transcriptional regulation of $f x k R$ was studied under aerobic and microaerobic conditions in the wild -type and in the hfixL and $f x k R$ mutants. Measurements of GUS activity in the wild-type strain revealed that $f x k R$ is expressed constitutively in both aerobic $(552 \pm 133)$ and microaerobic $(642 \pm 54)$ conditions. Similarly, levels of $f x k R$ expression remained unaltered in the $h$ fixL (aerobic $558 \pm 138$ and microaerobic $625 \pm 138$ ) and fxkR (aerobic $554 \pm 155$ and microaerobic $634 \pm 80$ ) mutants, regardless of the oxygen concentration in the culture. Therefore, it can be concluded that $f x k R$ is expressed constitutively under the tested conditions, and that FxkR does not regulate

Table 1. Identification of the response regulator that controls fixKf microaerobic expression in Rhizobium etli CFN42

\begin{tabular}{lllc}
\hline Strain & Gene mutated & \multicolumn{1}{c}{ Domain architecture and genetic organization } & $\begin{array}{c}\text { fixKf microaerobic expression } \\
\left.\text { (nmol min }^{-1} \mathbf{~ m g}^{-1} \text { of protein }\right)^{\mathbf{a}}\end{array}$ \\
\hline CE3 & & Wild type & $381 \pm 12$ \\
SR 72 & RHE_PF00072 & No HTH domain, with an additional nucleotide binding domain; orphan & $330 \pm 15$ \\
SR 224 & RHE_PF00224 & $\begin{array}{c}\text { No HTH domain, probably in an operon that includes one histidine-kinase (HK) } \\
\text { and two additional receiver domains }\end{array}$ & $292 \pm 22$ \\
SR 225 & RHE_PF00225 & NarL/FixJ superfamily, probably in the same operon as RHE_PF00224 & $282 \pm 17$ \\
SR 479 & RHE_PF00479 & NarL/FixJ superfamily, probably in operon with RHE_PF00480 (HK) & $289 \pm 18$ \\
SR 530 & RHE_PF00530 & OmpR/PhoB superfamily; orphan & $4 \pm 4$ \\
SR 533 & RHE_PF00533 & NarL/FixJ superfamily; orphan & $315 \pm 17$ \\
SR 547 & RHE_PF00547 & No HTH domain, probably in operon with RHE_PF00548 & $299 \pm 24$ \\
SR 548 & RHE_PF00548 & NarL/FixJ superfamily, probably in operon with RHE_PF00547 & $302 \pm 17$ \\
\hline
\end{tabular}

${ }^{a} \beta$-Glucuronidase-specific activity was determined after $10 \mathrm{~h}$ at $30^{\circ} \mathrm{C}$ under microaerobic conditions. Data are the mean of two replicas from three independent experiments.

Table 2. Microaerobic expression analysis of the Rhizobium etli fixKf::uidA transcriptional fusion in different genetic backgrounds

\begin{tabular}{lclc}
\hline Strain & Strain genotype & \multicolumn{1}{c}{ Plasmids } & Expression level (nmol min $^{\mathbf{- 1}} \mathbf{~ m g}^{\mathbf{- 1}}$ of protein) $^{\mathbf{a}}$ \\
\hline CE3/pLG1 & Wild type & pBBMCS53::fixKf & $367 \pm 20$ \\
CFNX636/pLG1 & fixL::loxPSp & pBBMCS53::fixKf & $2 \pm 2$ \\
CFN-LG1/pLG1 & fxkR::loxPSp & pBBMCS53::fixKf & $1 \pm 4$ \\
CFNX636/pLG1/pfxkR-Ret & fixL::loxPSp & pBBMCS53::fixKf pRK415::fxkR-Ret & $60 \pm 12$ \\
CFN-LG1/pLG1/pfxkR-Ret & fxkR ::loxPSp & pBBMCS53::fixKf pRK415::fxkR-Ret & $697 \pm 70$ \\
CFN-LG1/pLG1/pfxkR-VF & fxkR::loxPSp & pBBMCS53::fixKf pRK415::fxkR-Rleg & $510 \pm 71$ \\
CFN-LG1/pLG1/pfixJ-Sm & fxkRR::loxPSp & fixKf::uidA pRK415::fixJ-Smel & $6 \pm 5$ \\
CFN-LG1/pLG1/pRK415 & fxkR::loxPSp & pBBMCS53::fixKf pRK415 & $2 \pm 2$ \\
\hline
\end{tabular}

${ }^{a} \beta$-Glucuronidase-specific activity was determined after $10 \mathrm{~h}$ at $30^{\circ} \mathrm{C}$ under microaerobic conditions. Data are the mean of two replicas from two independent experiments. 
the expression of its own promoter. Considering that fix $\mathrm{Kf}$ expression is highly induced under microaerobiosis (25-fold) (Girard et al. 2000), we can propose that hFixL activates FxkR post-translationally in this condition.

\section{Plasmid pRL9 of $R$. leguminosarum bv. viciae 3841 harbors an $f x k R$-related gene.}

As mentioned earlier, $R$. etli CFN42 and R. leguminosarum bv. viciae share a novel hFixL and the absence of an annotated fixJ gene (Girard et al. 2000; Patschkowski et al. 1996). Given these similarities, it was considered probable that an $f x k R$-like gene would be present in $R$. leguminosarum bv. viciae. Sequence analysis by BLASTP (National Center for Biotechnology Information [NCBI] GenBank Database) revealed that gene pRL90026 of $R$. leguminosarum bv. viciae 3841 encodes a 220-amino-acid polypeptide with high similarity to FxkR of $R$. etli. Based on the pRL90026 nucleotide sequence, a 1,230-bplong polymerase chain reaction (PCR) fragment was synthesized from both $R$. leguminosarum bv. viciae 3841 and VF39 strains. Complete sequence analysis of the PCR product from strain VF39 revealed a complete ORF (accession number FR854234) with high identity to pRL90026 and RHE_PF530 (98 and $88 \%$, respectively). To determine whether this gene encodes a functional homolog of $R$. etli FxkR, a constitutive expression plasmid was generated by cloning the putative $R$. leguminosarum bv. viciae VF39 fxkR gene under the control of the lacZ promoter in the pRK415 plasmid vector ( $\mathrm{p} f x k R-\mathrm{VF})$. This plasmid was introduced into the $R$. etli fxkR mutant strain (CFN-LG1) containing the fixKf::uidA fusion. Complementation of the CFN-LG1 ( $f x k R: \because$ lox $\mathrm{Sp}$ ) mutant strain with plasmid $\mathrm{p} f x k R$-VF restored fixKf microaerobic expression to levels similar to those observed when the fxkR::loxSp mutant was complemented with its own $f x k R$ gene (Table 2, compare lines 1 and 3 with 5 and 6). These results indicate that $f x k R$ is a novel regulatory element, present in both $R$. etli CFN42 and $R$. leguminosarum bv. viciae, and is indispensable in activating fixKf transcription. We also tested the ability of fixJ from $S$. meliloti to restore expression of fixKf in the absence of FxkR in $R$. etli. As expected, this gene was unable to activate expression of $R$. etli fixKf (Table 2, line 7). This result is in agreement with the observation that the consensus binding sites of FixJ (Ferrières and Kahn 2002) are not conserved in the regulatory region of fixKf (data not shown). It is noteworthy that, even if there were less-effective binding sites for FixJ in the regulatory region of fixKf, hFixL might not be able to use FixJ from $S$. meliloti as its cognate response regulator.

\section{FxkR-related proteins are widely distributed in $\alpha$-proteobacteria.}

To identify the presence of FxkR- and hFixL-related proteins in other bacteria, a BLASTP search was performed using the $R$. etli CFN42 hFixL and FxkR proteins as query sequences. The proteins with the highest percentage of identity to $R$. etli CFN42 FxkR and hFixL belonged to bacteria from the rhizobial group (a list containing the complete BLASTP hits is reported in Supplementary Table S2). For further comparison analysis, we selected those bacteria that harbored both highly conserved hFixL and FxkR proteins and whose genomes were completely sequenced. To this end, hFixL proteins were selected on the basis of the presence of a C-terminal receiver domain, the conserved $\mathrm{His}^{200}$ and $\mathrm{Arg}^{220}$ that are important for oxygen binding in FixL from $B$. japonicum, and the presence of the unorthodox $\mathrm{H}$ box sequence HDFNNLL (Boesten and Priefer 2004; Dunham et al, 2003; Gong et al. 1998). Multiple sequence analysis of hFixL-related proteins is presented in Supplementary Figure S2. The FxkR-related proteins were selected considering a score alignment of $\geq 48$ defined by ClustalW2, the presence of key amino acids for the function of the OmpR regulator (Supplementary Fig. S3), and their genomic context. The schematic representation of the genomic context and the amino acid identity between the hFixL- and FxkR-related proteins when compared with the $R$. etli counterparts is shown in Figure 2. Interestingly, genomic context analysis revealed the presence of encoded FixKf-related proteins in all the organisms studied. Three types of arrangement defined by gene conservation or gene orientation were observed when the genomic context of $h f i x L$ - and $f x k R$-related genes was analyzed (Fig. $2 \mathrm{~A})$. The type 1 arrangement is characterized by the presence of known targets of the $R$. etli FixKf regulator; namely, the fixNOQP operon (Girard et al. 2000) and the azuPf gene (L. Girard, unpublished data). Also, it is noteworthy that $f x k R$ is not contiguous to $h$ fixL. The type 2 and 3 arrangements both have contiguous $f x k R$, fixK, and $h f i x L$ genes and differ only with respect to gene orientation (Fig. 2A). Remarkable aspects of this set of genes are further addressed in the discussion.

\section{The regulatory region}

of fixKf-related genes shares a common regulatory box.

The conserved genomic context of fixKf-related genes led us to propose that they might be similarly regulated. To identify common regulatory sequence-elements upstream of the structural genes, we used the web server MEME (Bailey and Elkan 1994). A conserved 20-bp motif with an E value of $1.6(10)^{-29}$ was discovered in the upstream region of all $11 \mathrm{fixKf}$-related genes. A graphic representation of the motif alignment, its position with respect to the translational start site, and the representation of the consensus sequence (Logo) are shown in Figure 3. The conserved motif is composed of two repetitive 6-bp subsites separated by four variable nucleotides (GTTACA-N $4^{-}$ GTTACA). The third position of the first subsite is the least conserved and the motifs found in the fix Kf f-related genes of Acidiphilum cryptum JF5 and Acidiphilum multivorum AIU301 present considerable variations with respect to the GTTACA$\mathrm{N}_{4}$-GTTACA consensus (Fig. 3A). This sequence highly resembles the F1 binding motif found in $o m p F$, which is bound with high affinity by the OmpR regulator (Yoshida et al. 2006). We named this conserved motif the $\mathrm{K}$ box (for fix Kf regulatory region) and the two subsites $\mathrm{Ka}$ and $\mathrm{Kb}$, respectively.

To determine the relevance of the $\mathrm{K}$ box for the activation of fixKf microaerobic expression, we designed an expression analysis. For this purpose, the transcriptional start site of $R$. etli fixKf was identified, by rapid amplification of cDNA (RACE) $5^{\prime}$ ends assays, using total RNA, purified from wildtype cells cultured under microaerobic conditions. Our results show that the fix Kf transcriptional start site is an A located 21 nucleotides upstream from the putative translational start codon. Analysis of the DNA region upstream of the fixKf gene revealed the presence of a purine-rich Shine-Dalgarno-like sequence (AGGGGA) seven bases in front of the fixKf ATG codon. Thus, the conserved motif (GTTACA-N $\mathrm{N}_{4}$ GTTACA) identified in this work is centered at position $-69 \mathrm{bp}$ from the fix $\mathrm{Kf}$ transcriptional start site (Fig. 4A). Different transcriptional fusions were constructed using DNA fragments containing deletions of the fixKf upstream sequence, synthesized by PCR, and fused with a promoterless GUS gene (Fig. 4A). The plasmid pDZS1 (fixKf::uidA $\mathrm{K} \mathrm{box}^{-}$) carries a PCR fragment of $528 \mathrm{bp}$ that does not include the K box; plasmid pDZS2 (fixKf::uidA $\mathrm{K}$ box) contains a shortened regulatory region (560 bp long) that begins with the $\mathrm{K}$ box; in pDZS3 (fixKf::uidA Ka-GTT), the first three nucleotides of the $\mathrm{Ka}$ subsite were deleted whereas, in pDZS4 (fixKf::uidA Ka-GTTACA ${ }^{-}$), the GTTACA sequence in $\mathrm{Ka}$ was deleted. Plasmids containing transcriptional fusions were introduced separately into the $R$. etli CE3 wild-type strain and their transcriptional activity was moni- 
tored under microaerobic conditions and compared with the expression pattern given by plasmid pLG1 (which contains the complete fixNf-fixKf intergenic region) (Fig. 4A).

The microaerobic expression of fixKf was drastically affected when the $\mathrm{K}$ box was deleted, indicating that this sequence is required (Fig. 4B compare the level of expression of pLG1 versus pDZS1). The level of microaerobic expression observed in the wild-type strain $(281 \pm 7)$ remained unaffected in pDZS2 (292 \pm 8 ), suggesting that the regulatory region present in this plasmid is sufficient for fixKf expression. A nearly $50 \%$ decrease in the expression of fixKf was observed when the first three nucleotides of the Ka subsite were deleted (pDZS3, $136 \pm$ 6 ), whereas deletion of the GTTACA sequence completely abolished transcription (pDZS4, $17 \pm 6$ ) (Fig. 4B). This result strongly suggests that the complete GTTACA region is an essential target site for fixKf activation. To evaluate the regula- tory role of each $\mathrm{Ka}$ and $\mathrm{Kb}$ subsite for the expression of fix $\mathrm{Kf}$, site-specific mutations were introduced in the regulatory region of fixKf present in the reporter fusion pLG1. The GTTACA motif of each subsite was substituted for an EcoRI restriction site individually in $\mathrm{pKa}^{*}$ or $\mathrm{pKb}$ *(from GTTACA to GAATTC) (Fig. 4A). It was found that microaerobic expression of fix $\mathrm{Kf}$ was drastically affected when either $\mathrm{Ka}$ or $\mathrm{Kb}$ motifs were mutated (17 \pm 8 and $11 \pm 2$, respectively) (Fig. 4B), confirming that the $\mathrm{K}$ box is crucial for fixKf activation and that both subsites are necessary for its correct function.

FxkR binds the fixNf-fixKf intergenic region in vitro.

To ascertain whether FxkR could directly interact with the fixKf regulatory region, gel shift assays were performed with an $R$. etli-purified recombinant $\mathrm{His}_{6}$-FxkR protein and a 300bp DNA fragment containing either the wild type or the mu-

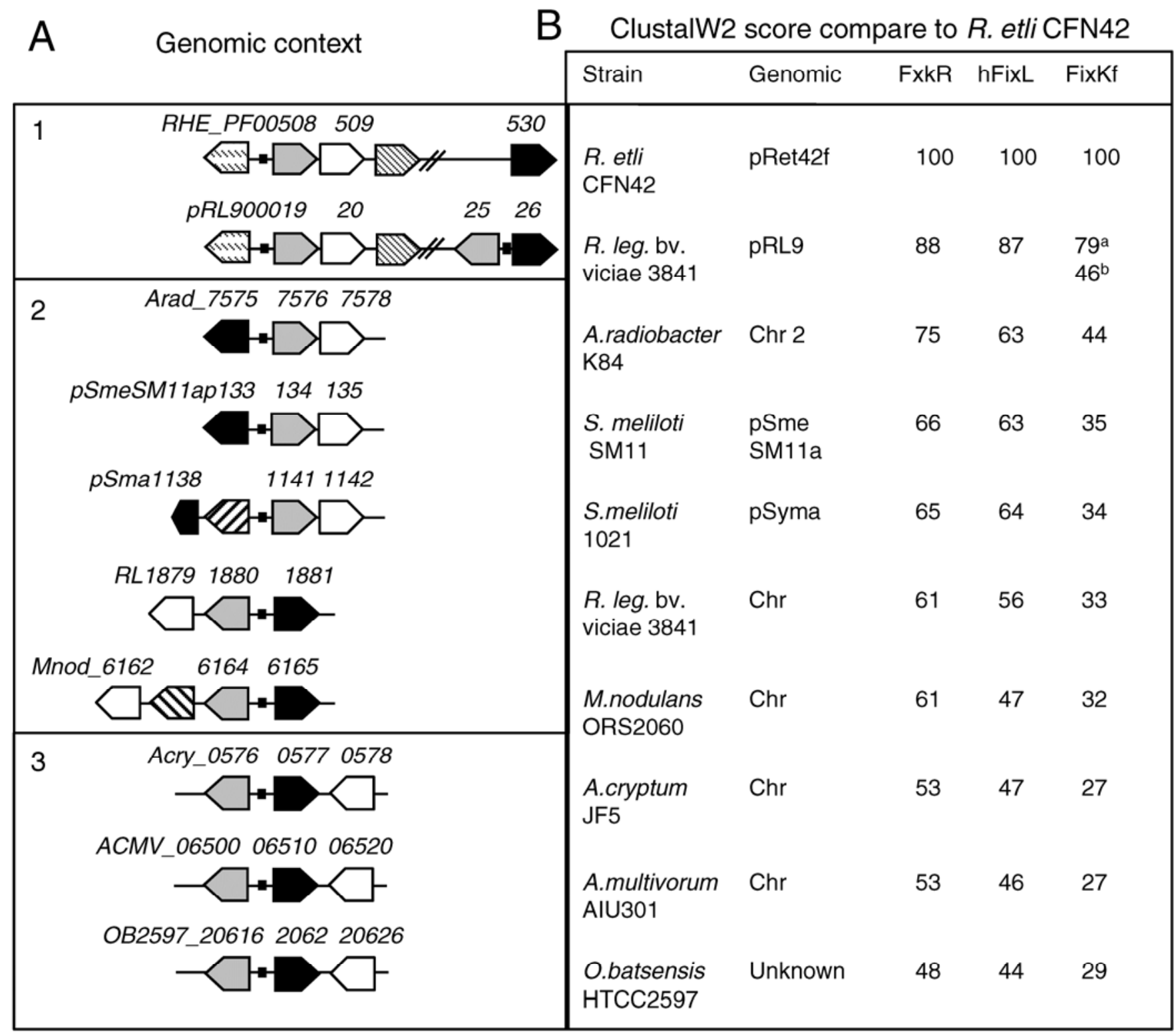

Fig. 2. Schematic representation of the genomic context and comparison analysis of fixKf-, $h$ fixL-, and $f x k R$-related genes in $\alpha$-proteobacteria. A, Schematic representation of the genomic context. Boxes 1,2, and 3 correspond to different types of arrangements identified. Similar arrangements are in the same box. Structural genes are represented by arrows that indicate the direction of transcription. fixKf-related genes are in gray, $h f i x L$-related genes in white, and fxkRrelated genes in black. The continuous line denotes genes encoded contiguously, while a broken line indicates that the genes are located separately. Genes that are within the hfixL-fixKf region are marked by a pattern that represents their identity (genes with the same pattern belong to the same protein family). The putative $\mathrm{K}$ boxes identified upstream from the fixK-related genes are indicated by black squares. B, ClustalW2 score of FxkR-, hFixL-, and FixKf-related proteins compared with the corresponding proteins in Rhizobium etli CFN42. Superscripts a and b correspond topRL90019 and pRL90025, respectively. 
tated $\mathrm{pKa}^{*}$ and $\mathrm{pKb}^{*}$ subsites (Fig. 5A). Incubation of the labeled wild-type probe with increasing concentrations of His $_{6}$-FxkR resulted in a higher amount of DNA-protein complex (Fig. 5B). Binding of this recombinant protein was specific, as judged by the fact that a 100-fold excess of unspecific unlabeled DNA (salmon sperm DNA) did not disrupt the FxkRtemplate complex whereas a 100-fold excess of unlabeled template displaced the labeled DNA in the complex (Fig. 5C, compare lanes 1 and 2). Additionally, competition assays revealed that the unlabeled mutated regions competed considerably less than the wild-type template, suggesting that FxkR is unable to bind to a region containing specific mutations in the $\mathrm{Ka}$ or $\mathrm{Kb}$ subsite ( $\mathrm{Ka}^{*}$ or $\mathrm{Kb}^{*}$, respectively) (Fig. $5 \mathrm{C}$, lanes 3 and 4). Furthermore, substitution of the $\mathrm{Ka}$ or $\mathrm{Kb}$ subsites with an EcoR1 restriction site $\left(\mathrm{Ka}^{*}\right.$ and $\left.\mathrm{Kb}^{*}\right)$ severely compromised binding of the $\mathrm{His}_{6}$-FxkR protein (Fig. 5D). These results permit us to conclude that FxkR is the direct activator of fix $\mathrm{Kf}$ expression, that the GTTACA sequence is the binding motif recognized by FxkR, and that both subsites are necessary for the correct binding of the regulator.

\section{DISCUSSION}

We have shown here that a response regulator that belongs to the OmpR/PhoB superfamily, herein named $f x k R$, is a key regulatory element that completes the hFixL-dependent regula- tory cascade for fix gene regulation. Our results demonstrate that FxkR is strictly necessary to activate fix $K \mathrm{f}$ expression in response to low oxygen concentration in $R$. etli CFN42, by binding to a specific sequence (GTTACA- $\mathrm{N}_{4}$-GTTACA) in the fix $K \mathrm{f}$ regulatory region ( $\mathrm{K}$ box) upon its activation. Because FxkR is the first transcriptional regulator reported to activate microaerobic expression of fix $\mathrm{Kf}$, this work casts light on a longstanding unanswered question of relevance in the field of nitrogen-fixation gene regulation.

Co-evolutionary adaptation has shaped the genetic circuits that control symbiosis and nitrogen fixation, leading to appreciable differences between unrelated symbiotic partners. The $R$. etli intricate regulatory cascade revealed a novel player, the response regulator FxkR. Notably, this response regulator is not related to the family of FixJ regulators that are widely accepted as cognate proteins of FixL sensors and the main distributors of the oxygen signal in several $\alpha$-proteobacteria.

It has been previously reported that mutations in either fixL or fix $K f$ do not affect nitrogen fixation. For that reason, it was not unexpected that the $R$. etli FxkR mutant showed an Fix ${ }^{+}$ phenotype (data not shown). Under symbiotic conditions, FnrN proteins are able to substitute for FixK to maintain nitrogen fixation in the bacteroids. The absence of a $\mathrm{K}$ box in the regulatory region of the $f n r N$ genes strongly suggests that these regulators are not under the control of FxkR in the nodule environment. The fact that the FnrN regulators were char-

A sequence name
----------
RHE_PF00508
pRL90025
pRL90019
SMa1141
pSmeSM11 ap134
Arad_7576
Mnod_6164
RL1880
ACMV_06500
ACry_0576
OB2597_20616

B

SEQUENCE NAME
P-value

--------

2.97e-12 ACGAACCGTC

7.66e-12 TCAAGCCGCG

$1.28 \mathrm{e}-11$ GCGAACCGCC

4.27e-11 CACTCCCAGC

4.27e-11 CACTCCCAGC

2.43e-10 CGGGCTGCGC

4.99e-10 ATCGGAAGAT

1.64e-09 GCATTCTCAA

6.55e-09 TTTATATGAA

6.55e-09 TTTATATGAA

1.34e-08 CCGGCGACAA

\section{Site}

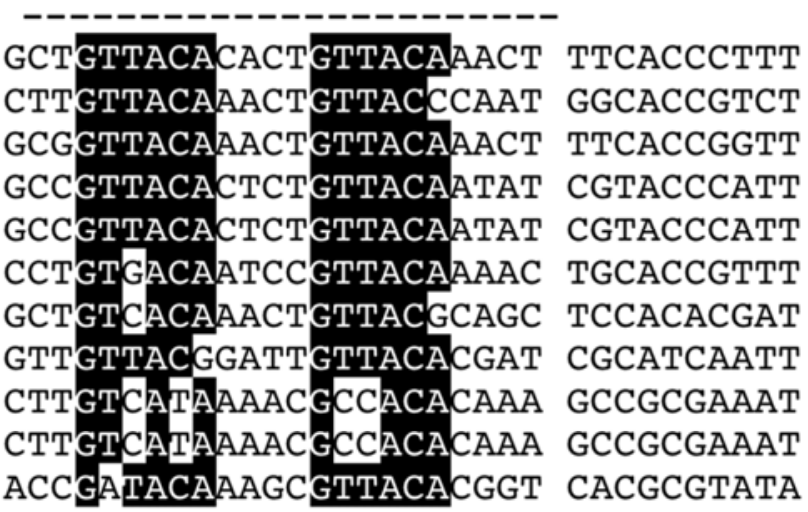

MOTIF LOCATION

C

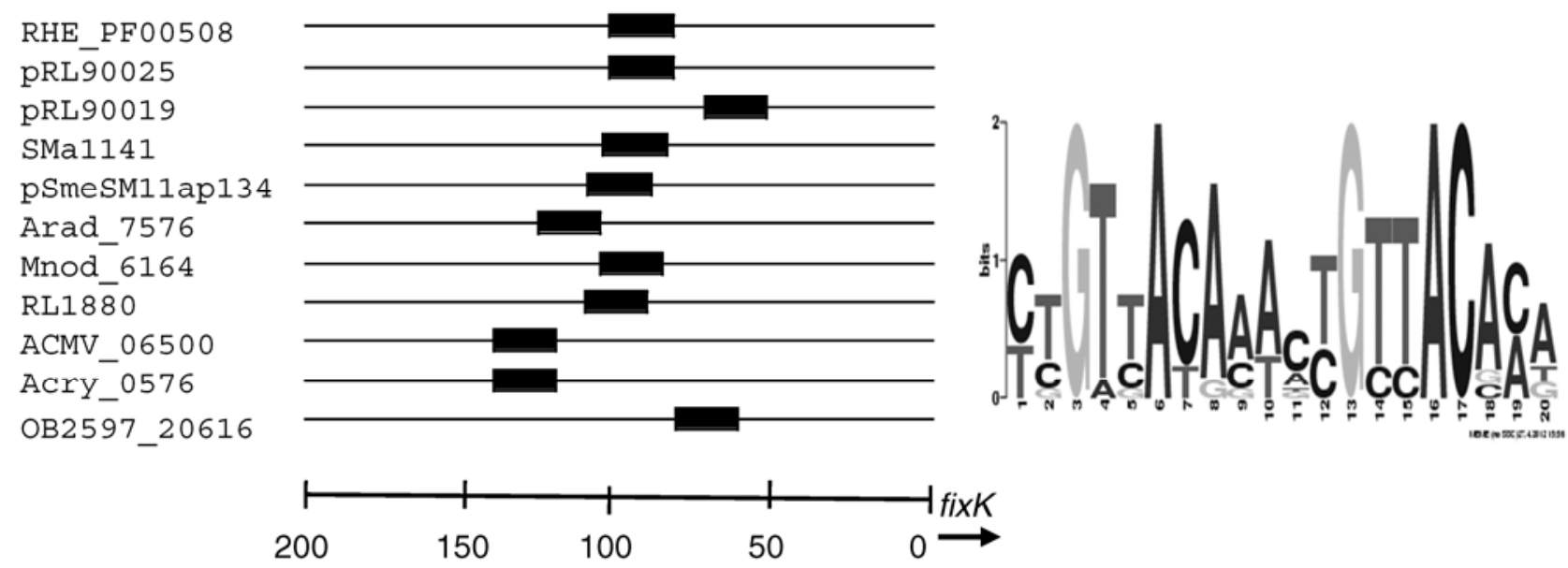

Fig. 3. Identification of the $\mathrm{K}$ box in the regulatory region of the fixKf-related genes. A, Sequence pattern and $P$ values found by MEME analyzing $200 \mathrm{bp}$ upstream of fixKf-related genes. Nucleotides in black boxes are the most conserved. B, Graphic representation of the conserved motif location with respect to the translational start of fixK. C, Logo representation that depicts the degree of conservation of the nucleotides along the discovered motif. 
acterized as oxygen sensors with the capability of autoactivation may explain their role as an independent branch in the control of nitrogen-fixing genes.

One of the aims of this work was to set the foundations for future work in other bacteria, which could share this novel regulatory element. For this purpose, we made an in silico search to find some conserved genetic tracts that include an $f x k R$ gene in related bacteria. hfixL and $f x k R$ genes were found in plasmids as well as in the chromosome of nitrogen-fixing and nonnitrogen-fixing $\alpha$-proteobacteria, not restricted to organisms that lack fixL and fixJ genes or have a fixNOQP operon. An open question would be whether these related genes have diverted to accomplish different physiological tasks. A closely related regulatory cascade was previously reported in $R$. leguminosarum bv. viciae VF39, which induces efficient symbiosis with legume plants belonging to the tribe Fabeae (Patschkowski et al. 1996). Here, we identified the presence of a highly conserved $f x k R$ gene in $R$. leguminosarum bv. viciae VF39 and demonstrated that this gene encoded for an FxkR protein able to restore the $R$. etli CFN42 fixKf gene expression in an $f x k R$ mutant. A promising perspective is to test whether this regulator controls the expression of fixK or fnrN in its own genetic background. Genome analysis of $R$. leguminosarum bv. viciae 3841 revealed the presence of a $\mathrm{K}$ box in the regulatory region of the three fixKf-related genes but not in that of fnrN. Therefore, one or all of these three genes might control expression of the symbiotic terminal oxidases directly or indirectly via $f n r N$. The regulatory scenario becomes more complex if we integrate the hFixL- and FxkR-related proteins encoded in the chromosome of this bacterium. Demonstration of the specificity of the FxkR regulators for the $\mathrm{K}$ boxes found upstream from different fix $K \mathrm{f}$-related genes and the specificity of the hFixL kinases for the different FxkR-related regulators could reveal whether these two-component systems are part of surefire or cross-talk circuits.

Using the available genomic information, we identified closely related hFixL, FxkR, and FixKf proteins in both nitrogen-fixing and non-fixing $\alpha$-proteobacteria (Fig. 2). Methylobacterium nodulans ORS2060 and S. meliloti SM11 have perhaps the most interesting gene set with respect to microaerobic response, due to the fact that, in addition to an FixL-FixJ-FixK regulatory cascade that controls expression of fixNOQP and fixGHIS operons, an hFixL-FxkR-FixK-related genetic locus is also present. It would be worth determining the participation of each regulator in the control of the microoxic-dependent expression and to learn which of these cascades control the expression of the different FNR/CRP regulators. Moreover, the fact that both types of FixL proteins (canonical and hybrid) coexists in the same cell raises the question of whether they detect the same stimulus and, if that is the case, what differences in the detecting threshold may exist. All these concerns should be addressed in order to better understand how similar regulatory pathways involved in signal transduction are orchestrated within the cell.

The presence of FixL-FixJ and hFixL-FxkR regulatory systems in non-nitrogen-fixing $\alpha$-proteobacteria implies a conserved modular design for oxygen sensing. Previous analyzes have described a correlation between the presence of this sen-

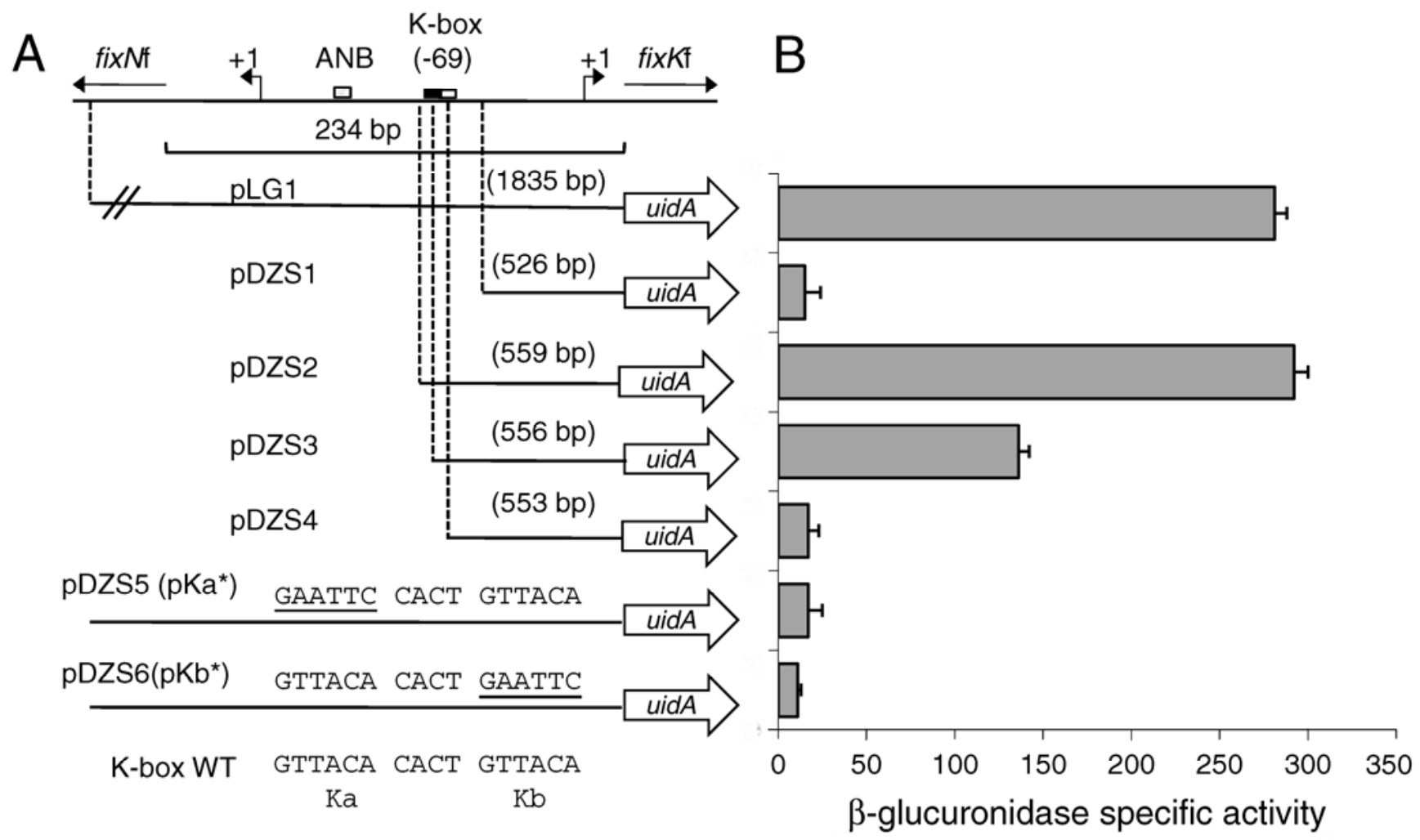

Fig. 4. Genetic analysis of the putative FxkR-binding site in the expression of Rhizobium etli fixKf. A, Schematic representation of the fixKf promoter region and fragments cloned in plasmid pBBMCS53 to generate fixKf-GUS transcriptional fusions. The fixNf and fixKf structural genes are represented by arrows that indicate transcription direction. The transcriptional start site of each gene is indicated by a +1 and by bent arrows. The $\mathrm{K}$ box is represented by a black or white box. The fixNf anaerobox is represented by an open box named ANB. Relative positions of the regulatory elements identified and the size of fragments cloned in plasmid pBBMCS53 are indicated. A scale representation of the length of the fusions is shown by dotted lines. Arrows at the end of each transcriptional fusion represent the structural uidA gene. The $\mathrm{pKa}^{*}$ and $\mathrm{pKb} \mathrm{b}^{*}$ legends denote the presence of site-directed mutations in the Ka and $\mathrm{Kb}$ subsites, respectively. Nucleotides underlined indicate introduced EcoRI site. B, Microaerobic expression analysis. Each bar of activity is positioned in accordance to the corresponding transcriptional fusion with respect to the graphical representation in A. GUS-specific activity is expressed as nanomoles of product per minute per milligram of protein. Data are the mean of two replicas from three independent experiments. 
sory systems and a ccoNOQP operon. In C. crescentus, the FixL-FixJ system also controls the expression of alternative cytochrome oxidases (Cosseau and Batut 2004; Crosson et al. 2005). Here, we report at least three different $\alpha$-proteobacteria that lack $c b b_{3}$ and possess an hFixL-FxkR sensory system (Acidiphilum cryptum, A. multivorum, and Agrobacterium radiobacter $\mathrm{K} 84$ ). These findings suggest to us that the utilization of this oxygen-responsive sensory system is not restricted to bacteria that have evolutionarily adapted to microaerobic environments by the presence of a high-affinity $c b b_{3}$ complex. Interestingly, in Acidiphilum cryptum and A. multivorum, we identified FixK-binding motifs (TTGAT-N ${ }_{4}$-ATCAA) in the regulatory region of genes implicated in different types of respiration processes such as $c y d A$ (involved in aerobic respiration), narG (important for nitrate-dependent respiration), or hem $N$ and $b c h E$ genes that form part of the tetrapyrrole pathway. These last two genes are involved in microaerobic respiration and photosynthesis in Rubrivivax gelatinosus (Ouchane et al. 2007). It is also significant that, in addition to FixK (Acry_0576 or ACMV_06500), a regulator of the FNR/CRP family with this conserved box is present. Both regulators may function in coordination to control the mentioned genes. In order to better understand oxygen-dependent adaptation among bacteria, it will be necessary to compare genes and proteins that are controlled in response to this signal.

Previous studies on the distribution of plasmid genetic markers in bean-nodulating strains revealed appreciable intraspecies diversity. Most of the Rhizobium etli strains tested showed the presence of a CFN42 hfixL homolog encoded in a plasmid different from the symbiotic one, and the absence of a fixJ gene (Brom et al. 2002). Using CFN42 fxkR- and hfixLspecific primers, we obtained the expected PCR products from $18 R$. etli strains. Partial sequence analysis from $12 \mathrm{f} x \mathrm{kR}$ products, including those from CFN42 and $R$. legumninosarum bv. viciae as controls, demonstrated that the products obtained correspond to $f x k R$ (L. Girard, unpublished data). This result supports the idea that the presence of hFixL and FxkR is predominant in $R$. etli strains and suggests that this regulatory circuit is common among these closely related bacteria. However, most of these strains were isolated from Spain or Mexico, and it would be interesting to know how conserved the hfixL and $f x k R$ genes are in a richer collection of $R$. etli strains isolated from different soil sources.

Despite the evident variability between the intergenic regions of fixkf genes, the regulatory box was highly but not completely conserved among them. The least-conserved $\mathrm{K}$ boxes with the lowest position $P$ value (Fig. 3A) were those of Acidiphilum cryptum, Acidiphilum multivorum, and Oceanicola batsensis HTCC2597. FxkR proteins from these bacteria also possessed an HTH domain with the lowest ClustalW2 score when compared with the domain of FxkR from $R$. etli. It is probable that the FxkR-related proteins in these bacteria bind a different kind of regulatory box. PhoB-binding sites (TGTCA and TTACA) have been identified in the pstS promoter in Escherichia coli (Makino et al. 1996). Interestingly, the TTACA box is also included in the $\mathrm{K}$ box but the TGTCA region is not, although it can be found in the intergenic regions of fixKf-related genes from Agrobacterium radiobacter, Methylobacterium nodulans, Acidiphilum cryptum, and Acidiphilum multivorum and in the fixKf-related gene encoded in the chromosome of $R$. leguminosarum (data not shown). The importance of these nucleotides remains to be shown.

From the evidence presented in this work, we propose that hFixL and FxkR are part of a novel phosphorelay system that activates transcription of fixKf-related genes in response to oxygen, not only in the Rhizobium genus but also in other groups of $\alpha$-proteobacteria. To better understand the role of phosphorylation in the regulatory mechanisms of this activator, further biochemical and genetic characterization will be crucial. In this direction, it will be necessary to know the role of the conserved aspartate target of phosphorylation in the ability to control binding affinity or the oligomeric state of the regulator. Because the two-component systems that involve hHK are characterized by a cascade of phosphate transfer reactions, where the activities of the histidine-kinase (HK), histidine phosphotransferase, and receiver domains (RR) are essential, experiments are underway in our laboratory to determine whether additional elements are necessary for the functionality of the hFixL-FxkR regulatory cascade.

\section{MATERIALS AND METHODS}

Bacterial strains, plasmids, and growth conditions.

The bacterial strains and plasmids used are listed in Table 3. E. coli strains were grown at $37^{\circ} \mathrm{C}$ in Luria-Bertani (LB) medium and the antibiotics were added at the following concentrations: carbenicillin, $100 \mu \mathrm{g} \mathrm{ml}{ }^{-1}$; gentamicin $(\mathrm{Gm}), 15 \mu \mathrm{g}$

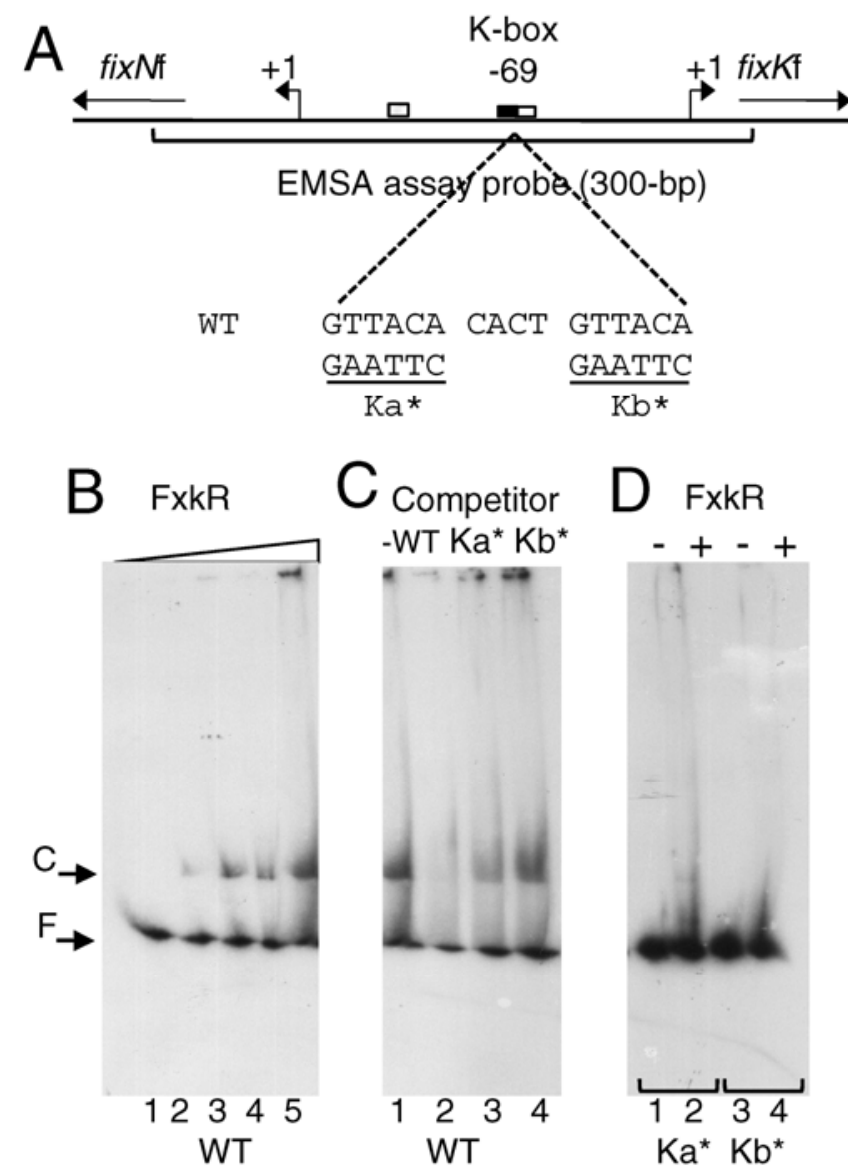

Fig. 5. Rhizobium etli FxkR fusion protein binds specifically to the K box. A, Graphic representation of the region used as probe for the electrophoretic mobility shift assays (EMSA). Wild-type probe and the substitution mutations indicated beneath (WT, $\mathrm{Ka}^{*}$, and $\mathrm{Kb}^{*}$, respectively). B, EMSA was performed without $\mathrm{His}_{6}$-FxkR protein (lane 1) or with 0.3 , $0.7,1.4$, and $2.9 \mathrm{nM}$ of $\mathrm{His}_{6}$-FxkR protein (lanes 2,3,4, and 5 respectively) plus end-labeled WT probe. C, Competition assay was performed with $2.9 \mathrm{nM}$ of $\mathrm{His}_{6}$-FxkR plus end-labeled WT probe without specific unlabeled competitor (lane 1) or with 100-fold excess of specific cold competitor: WT (lane 2), Ka* (lane 3), and $\mathrm{Kb}^{*}$ (lane 4). D, Binding assay using as probes fragments with mutations in either Ka (lanes 1 and 2) or $\mathrm{Kb}$ (lanes 3 and 4) subsites without or with $2.9 \mathrm{nM} \mathrm{His}_{6}$-FxkR, as indicated. The FxkR-DNA complex $(\mathrm{C} \rightarrow)$ and free DNA $(\mathrm{F} \rightarrow)$ are indicated. 
$\mathrm{ml}^{-1}$; kanamycin $(\mathrm{Km}), 30 \mu \mathrm{g} \mathrm{m} \mathrm{m}^{-1}$; and spectinomycin (Sp), $100 \mu \mathrm{g} \mathrm{ml}^{-1}$. Rhizobium strains were grown at $30^{\circ} \mathrm{C}$ in peptone-yeast (PY) rich medium (Noel et al. 1984) or in Y minimal medium (MMY) with succinate $(10 \mathrm{mM})$ and ammonium chloride $(10 \mathrm{mM})$ as carbon and nitrogen sources, respectively (Bravo and Mora 1988). Antibiotics were used at the following concentrations: streptomycin (Sm), $200 \mu \mathrm{g} \mathrm{ml} \mathrm{g}^{-1}$; Gm, $15 \mu \mathrm{g}$ $\mathrm{ml}^{-1} ; \mathrm{Km}, 30 \mu \mathrm{g} \mathrm{ml}^{-1}$; nalidixic acid, $20 \mu \mathrm{g} \mathrm{ml}^{-1}$; neomycin, 60 $\mu \mathrm{g} \mathrm{ml} \mathrm{m}^{-1}$, and $\mathrm{Sp}, 100 \mu \mathrm{g} \mathrm{ml^{-1 }}$. When required, sucrose was added at $12.5 \%(\mathrm{wt} / \mathrm{vol})$. For detection of $\beta$-galactosidase and GUS activity, 5-bromo-4-chloro-3-indolyl- $\beta$-D-galactoside and 5-bromo-4-chloro-3-indolyl- $\beta$-D-glucuronide, respectively, were used in plates at $20 \mu \mathrm{g} \mathrm{ml}^{-1}$. For growth under microaerobic conditions, cultures were grown to mid-exponential phase in PY medium. Cells were collected by centrifugation, washed with sterile minimal medium (MMY), and concentrated 100fold. MMY $(20 \mathrm{ml})$ containing $10 \mathrm{mM}$ succinate and $10 \mathrm{mM}$ ammonium chloride as carbon and nitrogen sources were inoculated with these suspensions at an initial optical density at $540 \mathrm{~nm}\left(\mathrm{OD}_{540}\right)$ of 0.05 . Cultures were incubated in $150-\mathrm{ml}$ bottles closed with an airtight stopper and flushed with several volumes of an oxygen/argon (1:99, vol/vol) mixture. Cultures were grown with shaking $(200 \mathrm{rpm})$ for $10 \mathrm{~h}$ at $30^{\circ} \mathrm{C}$ (GranadosBaeza et al. 2007). In experiments that included the CE3 deriva- tives cured of pRet42f (CFNX186 or CFNX218), cultures were inoculated at $\mathrm{OD}_{540}$ of 0.25 and the bottles, flushed with an oxygen/argon $(1: 99, \mathrm{vol} / \mathrm{vol})$ mixture, were incubated for $10 \mathrm{~h}$ at $30^{\circ} \mathrm{C}$ for microaerobic induction.

\section{Measurement of GUS activity.}

Aerobic and microaerobic cultures of $R$. etli strains harboring transcriptional fusions were grown as described previously (Girard et al. 1996). Quantitative GUS activity was determined on 1.0-ml culture samples using 4-nitrophenyl $\beta$-D-glucuronide as substrate, as described previously (Girard et al. 2000). Data were normalized to total cell protein concentration by the Lowry method over a second set of 1.0-ml samples. Specific activities are reported in nanomoles of product per minute per milligram of protein. Plasmid profiles of the samples used for GUS determinations were analyzed using a modified Eckhardt procedure (Hynes and McGregor 1990).

\section{PCR amplification.}

Specific PCR primers were designed using the Oligo 6.0 software and were purchased from Unidad de Síntesis Química IBtUNAM. Accuprime Taq DNA polymerase high fidelity (Invitrogen, Carlsbad, CA, U.S.A.) was used in PCR reactions with a cycling regime that includes a denaturing step at $94^{\circ} \mathrm{C}$ for $1 \mathrm{~min}$

Table 3. Bacterial strains and plasmids

\begin{tabular}{|c|c|c|}
\hline Strain or plasmid & Relevant characteristics $^{\mathbf{a}}$ & Reference \\
\hline \multicolumn{3}{|l|}{ Rhizobiales } \\
\hline CE3 & $\mathrm{Sm}^{\mathrm{r}}$ derivative of CFN42 & Noel et al. 1984 \\
\hline CFNX89 & CE3 derivative pRet $42 \mathrm{~d}^{-}$ & Brom et al. 1992 \\
\hline CFNX186 & CE3 derivative $\mathrm{pRet} 42 \mathrm{f}$ & Brom et al. 1992 \\
\hline CFNX218 & CE3 derivative pRet $42 \mathrm{a}^{-}, \mathrm{b}^{-}, \mathrm{c}^{-}, \mathrm{d}^{-}, \mathrm{e}_{\Delta}, \mathrm{f}$ & Brom et al. 2000 \\
\hline CFNX636 & CE3 derivative, fixL::loxPSp & Girard et al. 2000 \\
\hline R. leguminosarum bv. viciae VF39 & Wild type, $\mathrm{Sm}^{\mathrm{r}}$ & Hynes et al. 1988 \\
\hline R. leguminosarum bv. viciae 3841 & Wild type & Johnston and Beringer 1975 \\
\hline Sinorhizobium meliloti 1021 & Wild type & Meade et al. 1982 \\
\hline CFN-LG1 & CE3 derivative, $f x k R:: l o x P S p$ & This work \\
\hline \multicolumn{3}{|l|}{ Escherichia coli } \\
\hline DH5 $\alpha$ & 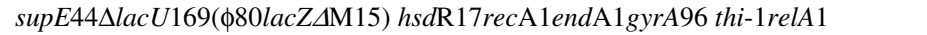 & Sambrook et al. 1989 \\
\hline BL21 (DE3) & F-ompT hsdS $S_{B}\left(r_{B-} m_{B-}\right)$ gal dcm (DE3) & Invitrogen \\
\hline \multicolumn{3}{|c|}{ - } \\
\hline pCR2.1 TOPO & Cloning vector for PCR products, $\mathrm{Ap}^{\mathrm{r}} \mathrm{Km}^{\mathrm{r}}$ & Invitrogen \\
\hline pK*mobsacB & Suicide cloning vector, $\mathrm{Km}^{\mathrm{r}}$ & Schäfer et al. 1994 \\
\hline pRK415 & Broad host range plasmid, $\mathrm{Tc}^{\mathrm{r}}$ & Keen et al. 1988 \\
\hline pET28a & Expression vector, His-Tag, $\mathrm{Km}^{\mathrm{r}}$ & Novagen \\
\hline pBBMCS53 & $\Delta \mathrm{placZ}$ pBBR1MCS-5 derivative, carrying the promoterless uid $\mathrm{A}$ gene, $\mathrm{Gm}^{\mathrm{r}}$ & Girard et al. 2000 \\
\hline pRK2073 & Conjugation helper plasmid, $\mathrm{Sm}^{\mathrm{r}} / \mathrm{Sp}^{\mathrm{r}}$ & Better and Helinski 1983 \\
\hline pRK2013 & Conjugation helper plasmid, $\mathrm{Km}^{\mathrm{r}}$ & Figurski and Helinski 1979 \\
\hline pLG1 & pBBMCS53 derivative fixKf::uidA & Girard et al. 2000 \\
\hline pARG1 & $\mathrm{pK}^{*}$ mobsacB derivative, $f x k R:: \operatorname{lox} \mathrm{PSp}$ & This work \\
\hline $\mathrm{p} f x k R$-Ret & pRK415 derivative carrying the $R$. etli f $x k R$ gene under the lac $Z$ promoter & This work \\
\hline $\mathrm{p} f x k R-\mathrm{VF}$ & $\begin{array}{l}\text { pRK415 derivative carrying the } R \text {. leguminosarum bv. viciae VF39 fxkR ortholog } \\
\text { under the lacZ promoter }\end{array}$ & This work \\
\hline $\mathrm{p} f i x J-\mathrm{Sm}$ & pRK415 derivative carrying the $S$. meliloti fixJ gene under the lacZ promoter & This work \\
\hline pARG2 & pBBMCS53 derivative $f x k R:: u i d \mathrm{~A}$ & This work \\
\hline pDZS1 & $\begin{array}{l}\text { pBBMCS53 derivative fixKf:: uidA transcriptional fusion containing } 459 \text { bp of } \\
\text { the fixKf CDS and } 67 \text { bp upstream FixKf ATG, without K box }\end{array}$ & This work \\
\hline pDZS2 & $\begin{array}{l}\text { pBBMCS53 derivative fixKf::uidA transcriptional fusion containing } 459 \text { bp of } \\
\text { the fixKf CDS and } 100 \text { bp upstream FixKf ATG, including the K box complete }\end{array}$ & This work \\
\hline pDZS3 & $\begin{array}{l}\text { pBBMCS53 derivative fixKf::uidA transcriptional fusion containing } 459 \text { bp of } \\
\text { the fixKf CDS and } 97 \text { bp upstream FixKf ATG with a deletion of the GTT in } \\
\text { the Ka subsite }\end{array}$ & This work \\
\hline pDZS4 & $\begin{array}{l}\text { pBBMCS53 derivative fixKf::uidA transcriptional fusion containing } 459 \text { bp of } \\
\text { the fixKf CDS and } 94 \text { bp upstream FixKf ATG without the Ka subsite }\end{array}$ & This work \\
\hline pDZS5 & $\begin{array}{l}\text { pBBMCS53 derivative fixKf- pKa*::uidA transcriptional fusion with specific } \\
\text { mutations in subsite Ka }\end{array}$ & This work \\
\hline pDZS6 & $\begin{array}{l}\text { pBBMCS53 derivative fixKf-Kb*::uidA transcriptional fusion with specific } \\
\text { mutations in subsite } \mathrm{Kb}\end{array}$ & This work \\
\hline pARG3 & pET28a derivative carrying the $R$. etli f $x k R$ gene & This work \\
\hline
\end{tabular}

${ }^{\mathrm{a}} \mathrm{Sm}^{\mathrm{r}}, \mathrm{Ap}^{\mathrm{r}}, \mathrm{Km}^{\mathrm{r}}, \mathrm{Tc}^{\mathrm{r}}, \mathrm{Gm}^{\mathrm{r}}$, and $\mathrm{Sp}^{\mathrm{r}}$ indicate resistant to streptomycin, ampicillin, kanamycin, tetracycline, gentamicin, and spectinomycin, respectively; PCR = polymerase chain reaction. 
followed by 30 cycles of $94^{\circ} \mathrm{C}$ for $30 \mathrm{~s}, 45$ to $60^{\circ} \mathrm{C}$ (according the primer's melting temperature) for $30 \mathrm{~s}$, and $72^{\circ} \mathrm{C}$ for $90 \mathrm{~s}$. A final elongation step was made at $72^{\circ} \mathrm{C}$ for 5 to $10 \mathrm{~min}$, according to the length of the DNA fragment to be amplified.

\section{5' RACE.}

Transcriptional start sites for the fixKf and fixNf genes were identified in assays using the 5' RACE system for rapid amplification of cDNA ends (version 2.0; Invitrogen), as described previously (Gómez-Hernández et al. 2011). scDNA was synthesized from $2 \mu \mathrm{g}$ of total RNA purified from microaerobic cultures of the wild-type strain derivatives carrying fix $\mathrm{Kf}$ and fixNf transcriptional fusions in plasmid pBBMCS53 (pLG1 and pLG2, respectively) (Girard et al. 2000), using complementary primer gusLw. After first-strand cDNA synthesis, the mRNA was removed by treatment with RNase and the scDNA was purified using a SNAP column (Invitrogen). Terminal deoxynucleotidyl transferase and dCTP were used to add homopolymeric tails to the $3^{\prime}$ ends of the cDNA. Nested PCR reactions were done using gene specific primers fixKf-RT ( $5^{\prime}$ GGCGAAATGAAATGCGGTTAT-3', in positions 214 to 234 ) and fixNf-RT (5'-CGCTGCACCACGTAGAAC-3', in positions 403 to 420 ) and the abridged anchor primer (GGCCACGCGT CGACTAGTACGGGIIGGGIIGGGIIG, provided in the kit). The products were cloned into the pCR2.1 TOPO vector for sequencing.

\section{Plasmid construction.}

To introduce a mutation into the $f x k R$ gene, a 1,578-bp product was obtained by PCR using total DNA from strain CE3 and the specific primers UpDR530 and LwDR530. This PCR product was cloned by T-A annealing into pCR 2.1-TOPO (Invitrogen) and analyzed by sequencing. To construct a suicide plasmid useful for homogenotization (pARG-1), the 1,517-bp BglIIHindIII fragment containing the $f x k R$ allele was cloned into the BamHI-HindIII sites in pK*mobsacB (Schäfer et al. 1994). Mutagenesis of the $f x k R$ gene was achieved by introducing into the SalI site (nucleotide 274) a loxPSp interposon (Martínez-Salazar and Romero 2000). Plasmids for mutagenesis by vector-insertion (VIM) were constructed cloning a specific intragenic PCR product of the desired gene into the $\mathrm{pK}^{*}$ mobsacB vector, a $R h i$ zobium suicide plasmid. Intragenic regions were obtained by PCR using total DNA from strain CE3 and specific primers (primers and specific intragenic regions used for VIM are shown in Supplementary Table S1). These PCR products were cloned by T-A annealing into pCR 2.1-TOPO (Invitrogen) and analyzed by sequencing. To construct a suicide plasmid useful for VIM, the intragenic regions were purified using the EcoRI sites of the vector, with the exception of fragments for pPF530 and pPF72 that contain an internal EcoR1 site, and cloned into the pK*mobsacB (Schäfer et al. 1994).

Plasmids carrying the $R$. etli CFN42 fxkR (pfxkR-Ret), R. leguminosarum bv. viciae VF39 fxkR ( $\mathrm{p} f x k R-\mathrm{VF})$, and $S$. meliloti 1021 fixJ (pfixJ-Sm) genes constitutively expressed from the lac $Z$ promoter were obtained by cloning the respective coding region in the conjugative plasmid pRK415 (Keen et al. 1988). To construct $\mathrm{p} f x k R$-Ret, a 981-bp product was obtained by PCR using total DNA from strain CE3 and the specific primers Up530-HindIII and Lw530-XhoI. To generate plasmid pf $x k R$ VF, a 1,230-bp PCR product was obtained by PCR using total DNA from strain VF39 and the specific primers, based on the pRL90026 nucleotide sequence, UpRL9-26, and LwRL9-26. The fixJ gene of $S$. meliloti 1021 was obtained by PCR amplification using specific primers Up-fxJSm and Lw-fxJSm. To generate a plasmid for expression analysis of the $R$. etli fxkR gene, the corresponding regulatory region was synthesized by PCR using primers UP-530gus and Lw-PF530. An EcoRI frag- ment of 767 bp (containing 262 bp upstream from the FxkR start codon) was cloned in the broad-host plasmid pBBRMCS53 that carries a promoterless uidA gene (Girard et al. 2000), giving plasmid pARG-2. The correct orientation of the fragments in the plasmids was verified by PCR using primer gusLw and the corresponding upper primer in each case.

Plasmids pDZS1, pDZS2, pDZS3, and pDZS4 are shortened variants of plasmid pLG-1. Four different PCR products were obtained using plasmid pLG-1 as template and the specific forward primers Up-NA, Up-GTT, Up-ACA, and Up-CAC and the same reverse primer Lw-RRKf. The amplification products were cloned by T-A annealing into pCR 2.1-TOPO and sequenced. Fragments of 526 bp (containing 67 bp upstream from the FixKf start codon), 559 bp (containing 100 bp upstream from the FixKf start codon), 556 bp (containing $97 \mathrm{bp}$ upstream from the FixKf start codon), and 553 bp (containing 94 bp upstream from the FixKf start codon) were cloned separately in pBBMCS53, giving plasmids pDZS1, pDZS2, pDZS3, and pDZS4. The correct orientation of the fragments in the plasmids was verified by PCR using primer gusLw and the corresponding upper primer in each case. Mutations of the putative $\mathrm{K}$ box in fixKf promoter region were introduced using the QuikChange site-directed mutagenesis kit (Stratagene, La Jolla, CA, U.S.A.). Each subsite was modified by introducing an EcoRI site instead of the wild-type sequence (GTTACA). The primers used to mutate the Ka subsite were UpKa-mut and LwKa-mut. To mutate the $\mathrm{Kb}$ subsite, we used primers UpKb-mut and LwKb-mut. Underlined nucleotides in each primer denote the EcoR1 site introduced. Both modified fix $K \mathrm{f}$ regulatory regions were cloned in plasmid pBBMCS53 to obtain plasmids pDZS5 and pDZS6.

To generate a recombinant FxkR protein with a 6-histidine tag, the structural $f x k R$ region was amplified by PCR using total DNA from strain CE3 as template with the oligonucleotides UpFxkR-NdeI and Lw530-XhoI . The NdeI and XhoI sites introduced by the primers were used to clone the fragment into the pET28a vector to obtain pARG3. Primers used are described in the Supplementary Table S3.

\section{R. etli mutant derivatives.}

Mutagenesis by vector insertion was done using plasmids pPF72, pPF224, pPF225, pPF479, pPF530, pPF533, pPF547 and pPF547. Each plasmid, transferred by conjugation to Rhizobium spp., was co-integrated by single recombination with the homologous target, producing a knockout of the gene of interest (Landeta et al. 2011). Mutants were selected as resistant to $\mathrm{Sm}, \mathrm{Sp}$, and $\mathrm{Km}$ and the correct integration of the plasmid was verified by Southern blot hybridization.

Replacement of the $R$. etli CFN42 $f x k R$ wild-type allele by the f $x k R:: 1$ oxPSp mutant allele in plasmid pARG1 was carried out by homogenotization using the $s a c B$ marker present in the $\mathrm{pK}^{*}$ mobsacB vector; this marker allows an easy selection for homogenotization events, by scoring for resistance to sucrose. Double recombinants were selected as resistant to $\mathrm{Sm}$ and $\mathrm{Sp}$ and susceptible to $\mathrm{Km}$. To verify that the gene replacement had occurred, both plasmid profiles as well as total DNA blots of the derivatives were analyzed by Southern hybridization against the appropriate gene and cassette probes.

\section{Purification of $\mathrm{His}_{\mathbf{6}}$-FxkR.}

Plasmid pARG3 was used to overexpress the recombinant His $_{6}$-FxkR in the genetic background of E. coli BL21 (DE3). Overexpression was achieved by induction of cultures grown in $\mathrm{LB}$ media $\left(\mathrm{OD}_{540}=0.6\right)$ with $0.3 \mathrm{mM}$ isopropyl-thio-galactopyranoside for $2 \mathrm{~h}$ at $30^{\circ} \mathrm{C}$. Cells were collected by centrifugation at $4^{\circ} \mathrm{C}$ and lysed in native lysis buffer $\left(50 \mathrm{mM} \mathrm{NaH}{ }_{2} \mathrm{PO}_{4}\right.$ and $300 \mathrm{mM} \mathrm{NaCl}, \mathrm{pH} \mathrm{8.0)}$ after at least two passes through a 
French press. Separation of the soluble fraction was done after centrifugation at $10,000 \times g$ for $30 \mathrm{~min}$ at $4^{\circ} \mathrm{C}$. Samples of uninduced and induced cultures as well as of the soluble and insoluble fractions were analyzed by sodium dodecyl sulfate polyacrylamide gel electrophoresis (SDS-PAGE). His ${ }_{6}$-FxkR was purified from $5 \mathrm{ml}$ of the soluble fraction using Ni-NTA agarose (Invitrogen) in plastic columns (Bio-Rad, Hercules, CA, U.S.A.) following the instructions of the manufacturer. Briefly, the column was washed three times with washing buffer $\left(50 \mathrm{mM} \mathrm{NaH} \mathrm{PO}_{4}, 300 \mathrm{mM} \mathrm{NaCl}\right.$, and $40 \mathrm{mM}$ imidazol; $\mathrm{pH} 8.0$ ), and 16 fractions were collected after addition of elution buffer $\left(50 \mathrm{mM} \mathrm{NaH}_{2} \mathrm{PO}_{4}, 300 \mathrm{mM} \mathrm{NaCl}\right.$, and $500 \mathrm{mM}$ imidazole; $\mathrm{pH}$ 8.0). Samples of each purification step were analyzed by SDS-PAGE.

\section{Electrophoretic mobility shift assays.}

A 300-bp region encompassing the intergenic region between fixNf and fixKf was amplified by PCR using, as template, $\mathrm{pLG}-1$ or pDSZ-5 or pDSZ-6 with primers Up-RRKf and Lw2-RRKf. The PCR products were purified with the PureLink PCR purification kit (Invitrogen) and quantified with a Nanodrop spectrophotometer. DNA (100 ng) was used in the labeling reaction with $3 \mu \mathrm{l}$ of $\left[\gamma^{32} \mathrm{P}\right]$-ATP at $6,000 \mathrm{Ci} / \mathrm{mmol}$ in a final volume of $20 \mathrm{ml}$. Unincorporated label was removed with illustra Microspin G-25 columns (Amersham, Bucks, U.K.) following the instructions of the manufacturer.

For the binding reactions, $1 \mathrm{ng}$ of labeled DNA was incubated with an increasing concentration of pure protein $(0.3$ to $1.4 \mathrm{nM})$ in a binding buffer containing $100 \mathrm{mM}$ Tris- $\mathrm{HCl}(\mathrm{pH}$ 7.4), $100 \mathrm{mM} \mathrm{KCl}, 10 \mathrm{mM} \mathrm{MgCl} 2,10 \%$ glycerol, $2 \mathrm{mM}$ dithiothreitol, and $1 \mathrm{mM}$ lithium potassium acetyl phosphate (SigmaAldrich, St. Louis) in a final volume of $20 \mu \mathrm{l}$ for $20 \mathrm{~min}$ at room temperature. When indicated, $100 \mathrm{ng}$ of salmon sperm DNA or $100 \mathrm{ng}$ of unlabeled DNA was used as nonspecific or specific competitors. The electrophoresis run was carried on a $5 \%$ polyacrylamide $(79: 1)$ gel in $0.5 \times$ Tris-borate-EDTA buffer for $1 \mathrm{~h}$ at $300 \mathrm{~V}$ at room temperature.

\section{Bioinformatics resources.}

BLAST (blastp) was used to identify similar FxkR proteins in related organisms. The genomic context of the $f x k R$ genes was analyzed with the gene information viewer of the NCBI web page. Sequence alignment for the analysis of conserved residues and percentage of identity was performed with default parameters of the web server CLUSTAL2 of EMBL-EBI bioinformatic tools (Larkin et al. 2007). Domain conservation was analyzed using different domain libraries such as INTERPRO, PFAM, PROSITE, SMART, and SUPERFAMILY (Finn et al. 2010; Gasteiger et al. 2003; Letunic et al. 2009; Sigrist et al. 2010).

\section{ACKNOWLEDGMENTS}

We thank J. Mora for his support and stimulating discussions, M. Dunn for critical review of the manuscript, R. M. Ocampo and P. Salas for skillful technical assistance, Y. Mora for nitrogen fixation experiments, S. Brom and L. Cervantes for providing some Rhizobium strains, and P. Gaytán and E. López for oligonucleotide synthesis. Partial financial support was provided by PAPIIT-UNAM (grants IN202109 and IN219709) and CONACYT (grants 152776 and 80684). D. Zamorano-Sánchez was supported during the Ph.D. program (Programa de Doctorado en Ciencias Biomédicas, Universidad Nacional Autónoma de México) by a scholarship from Consejo Nacional de Ciencia y Tecnología (México).

\section{LITERATURE CITED}

Anthamatten, D., and Hennecke, H. 1991. The regulatory status of the fixL-like and fixJ-like genes in Bradyrhizobium japonicum may be different from that in Rhizobium meliloti. Mol. Gen. Genet. 225:38-48.
Anthamatten, D., Scherb, B., and Hennecke, H. 1992. Characterization of a fixLJ-regulated Bradyrhizobium japonicum gene sharing similarity with the Escherichia coli fnr and Rhizobium meliloti fixK genes. J. Bacteriol. 174:2111-2120.

Bailey, T. L., and Elkan, C. 1994 Fitting a mixture model by expectation maximization to discover motifs in biopolymers. Proc. Int. Conf. Intell. Syst. Mol. Biol. 2:28-36

Barakat, M., Ortet, P., and Whitworth, D. E. 2011. P2CS: a database of prokaryotic two-component systems. Nucleic Acids Res. 39:D771D776.

Batut, J., Daveran-Mingot, M. L., David, M., Jacobs, J., Garnerone, A. M., and Kahn, D. 1989. fixK, a gene homologous with fnr and crp from Escherichia coli, regulates nitrogen fixation genes both positively and negatively in Rhizobium meliloti. EMBO (Eur. Mol. Biol. Organ.) J. 8:1279-1286.

Bauer, E., Kaspar, T., Fischer, H. M., and Hennecke, H. 1998. Expression of the fixR-nifA operon in Bradyrhizobium japonicum depends on a new response regulator, RegR. J. Bacteriol. 180:3853-3863.

Better, M., and Helinski, D. P. 1983. Isolation and characterization of a recA gene of Rhizobium meliloti. J. Bacteriol. 115:311-316.

Boesten, B., and Priefer, U. B. 2004. The C-terminal receiver domain of the Rhizobium leguminosarum bv. viciae FixL protein is required for free-living microaerobic induction of the $f n r N$ promoter. Microbiology 150:3703-3713.

Bose, A., and Newman, D. K. 2011. Regulation of the phototrophic iron oxidation (pio) genes in Rhodopseudomonas palustris TIE-1 is mediated by the global regulator, FixK. Mol. Microbiol. 79:63-75.

Bravo, A., and Mora, J. 1988. Ammonium assimilation in Rhizobium phaseoli by the glutamine synthetase-glutamate synthase pathway. J. Bacteriol. 170:980-984.

Britos, L., Abeliuk, E., Taverner, T., Lipton, M., McAdams, H., and Shapiro, L. 2011. Regulatory response to carbon starvation in Caulobacter cres centus. PLoS One 6:e18179.

Brom, S., García de los Santos, A., Stepkowsky, T., Flores, M., Dávila, G., Romero, D., and Palacios, R. 1992. Different plasmids of Rhizobium leguminosarum bv. phaseoli are required for optimal symbiotic performance. J. Bacteriol. 174:5183-5189.

Brom, S., García-de los Santos, A., Cervantes, L., Palacios, R., and Romero, D. 2000. In Rhizobium etli symbiotic plasmid transfer, nodulation competitivity and cellular growth require interaction among different replicons. Plasmid 44:34-43.

Brom, S., Girard, L., García-de los Santos, A., Sanjuan-Pinilla, J. M., Olivares, J., and Sanjuan, J. 2002. Conservation of plasmid-encoded traits among bean-nodulating Rhizobium species. Appl. Environ. Microbiol. 68:2555-2561.

Cosseau, C., and Batut, J. 2004. Genomics of the $c c o N O Q P$-encoded $c b b_{3}$ oxidase complex in bacteria. Arch. Microbiol. 181:89-96.

Crosson, S., McGrath, P. T., Stephens, C., McAdams, H. H., and Shapiro, L. 2005. Conserved modular design of an oxygen sensory/signaling network with species-specific output. Proc. Natl. Acad. Sci. U.S.A. 102:8018-8023.

David, M., Daveran, M. L., Batut, J., Dedieu, A., Domergue, O., Ghai, J. Hertig, C., Boistard, P., and Kahn, D. 1988. Cascade regulation of nif gene expression in Rhizobium meliloti. Cell 54:671-683.

Dixon, R., and Kahn, D. 2004. Genetic regulation of biological nitrogen fixation. Nat. Rev. Microbiol. 2:621-631.

Dunham, C. M., Dioum, E. M., Tuckerman, J. R., Gonzalez, G., Scott, W. G., and Gilles-Gonzalez, M. A. 2003. A distal arginine in oxygen-sensing heme-PAS domains is essential to ligand binding, signal transduction, and structure. Biochemistry 42:7701-7708.

Ferrières, L., and Kahn, D. 2002. Two distinct classes of FixJ binding sites defined by in vitro selection. FEBS (Fed. Eur. Biochem. Soc.) Lett. 517:185-189

Figurski, D. H., and Helinski, D. R. 1979. Replication of an origin-containing derivative of plasmid RK2 dependent on a plasmid function provided in trans. Proc. Natl. Acad. Sci. U.S.A. 76:1648-1652.

Finn, R. D., Mistry, J., Tate, J., Coggill, P., Heger, A., Pollington, J. E., Gavin, O. L., Gunasekaran, P., Ceric, G., Forslund, K., Holm, L., Sonnhammer, E. L., Eddy, S. R., and Bateman, A. 2010. The Pfam protein families database. Nucleic Acids Res. 38:D211-D222.

Fischer, H. M. 1994. Genetic regulation of nitrogen fixation in rhizobia. Microbiol. Rev. 58:352-386.

Gasteiger, E., Gattiker, A., Hoogland, C., Ivanyi, I., Appel, R. D., and Bairoch, A. 2003. ExPASy: the proteomics server for in-depth protein knowledge and analysis. Nucleic Acids Res. 31:3784-3788.

Gilles-Gonzalez, M. A., and Gonzalez, G. 1993. Regulation of the kinase activity of heme protein FixL from the two-component system FixL/FixJ of Rhizobium meliloti. J. Biol. Chem. 268:16293-16297.

Girard, L., Valderrama, B., Palacios, R., Romero, D., and Dávila, G. 1996. Transcriptional activity of the symbiotic plasmid of Rhizobium etli is 
affected by different environmental conditions. Microbiology 142:26472856.

Girard, L., Brom, S., Dávalos, A., López, O., Soberón, M., and Romero, D. 2000. Differential regulation of fixN-reiterated genes in Rhizobium etli by a novel fixL-fixK cascade. Mol. Plant-Microbe Interact. 13:12831292.

Gómez-Hernández, N., Reyes-González, A., Sánchez, C., Mora, Y., Delgado, M. J, and Girard, L. 2011. Regulation and symbiotic role of nirK and norC expression in Rhizobium etli. Mol. Plant-Microbe Interact. 24:233-245.

Gong, W., Hao, B., Mansy, S. S., Gonzalez, G., Gilles-Gonzalez, M. A., and Chan, M. K. 1998. Structure of a biological oxygen sensor: a new mechanism for heme-driven signal transduction. Proc. Natl. Acad. Sci. U.S.A. 95:15177-15182.

González, V., Bustos, P., Ramírez-Romero, M. A., Medrano-Soto, A., Salgado, H., Hernández-González, I., Hernández-Celis, J. C., Quintero, V., Moreno-Hagelsieb, G., Girard, L., Rodríguez, O., Flores, M., Cevallos, M. A., Collado-Vides, J., Romero, D., and Dávila, G. 2003. The mosaic structure of the symbiotic plasmid of Rhizobium etli CFN42 and its relation to other symbiotic genome compartments. Genome Biol. 4:R36.

González, V., Santamaría, R. I., Bustos, P., Hernández-González, I., Medrano-Soto, A., Moreno-Hagelsieb, G., Janga, S.C., Ramírez, M. A., Jiménez-Jacinto, V., Collado-Vides, J., and Dávila, G. 2006. The partitioned Rhizobium etli genome: genetic and metabolic redundancy in seven interacting replicons. Proc. Natl. Acad. Sci. U.S.A. 103:3834-3839.

Granados-Baeza, M. J., Gómez-Hernández, N., Mora, Y., Delgado, M. J., Romero, D., and Girard, L. 2007. Novel reiterated Fnr-type proteins control the production of the symbiotic terminal oxidase $c b b_{3}$ in Rhizobium etli CFN42. Mol. Plant-Microbe Interact. 20:1241-1249.

Hynes, M. F., and McGregor, N. F. 1990. Two plasmids other than the nodulation plasmid are necessary for formation of nitrogen-fixing nodules by Rhizobium leguminosarum. Mol. Microbiol. 4:567-574.

Hynes, M. F., Brucksch, K., and Priefer, U. 1988. Melanin production encoded by a cryptic plasmid in a Rhizobium leguminosarum strain. Arch. Microbiol. 150:326-332.

Johnston, A. W., and Beringer, J. E. 1975. Identification of the Rhizobium 749 strains in pea root nodules using genetic markers. J. Gen. Microbiol. 87:343-350.

Kaminski, P. A., Batut, J., and Boistard, P. 1998. A survey of symbiotic nitrogen fixation by Rhizobia. Pages 431-460 in: The Rhizobiaceae: Molecular Biology of Model Plant-Associated Bacteria. H. P. Spaink, A. Kondorosi, and P. J. J. Hooykaas, eds. Kluwer Academic Publishers, Dordrecht, The Netherlands.

Keen, N. T., Tamaki, S., Kobayashi, D., and Trollinger, D. 1988. Improved broad-host-range plasmids for DNA cloning in gram-negative bacteria. Gene 70:191-197.

Landeta, C., Dávalos, A., Cevallos, M. A., Geiger, O., Brom, S., and Romero, D. 2011. Plasmids with a chromosome-like role in rhizobia. J. Bacteriol. 193:1317-1326.

Larkin, M. A., Blackshields, G., Brown, N. P., Chenna, R., McGettigan, P. A., McWilliam, H., Valentin, F., Wallace, I. M., Wilm, A., Lopez, R., Thompson, J. D., Gibson, T. J., and Higgins, D. G. 2007. Clustal W and Clustal X version 2.0. Bioinformatics 23:2947-2948.

Letunic, I., Doerks, T., and Bork, P. 2009. SMART 6: recent updates and new developments. Nucleic Acids Res. 37:D229-232.

Lopez, O., Morera, C., Miranda-Rios, J., Girard, L., Romero, D., and Soberón, M. 2001. Regulation of gene expression in response to oxygen in Rhizobium etli: role of FnrN in fixNOQP expression and in symbiotic nitrogen fixation. J. Bacteriol. 183:6999-7006.

Makino, K., Amemura, M., Kawamoto, T., Kimura, S., Shinagawa, H., Nakata, A., and Suzuki, M. 1996. DNA binding of PhoB and its interaction with RNA polymerase. J. Mol. Biol. 259:15-26.

Martínez-Salazar, J. M., and Romero, D. 2000. Role of the ruvB gene in homologous and homeologous recombination in Rhizobium etli. Gene
243:125-131.

Meade, H. M., Long, S. R., Ruvkun, G. B., Brown, S. E., and Ausubel, F. M. 1982. Physical and genetic characterization of symbiotic and auxotrophic mutants of Rhizobium meliloti induced by transposon Tn5 mutagenesis. J. Bacteriol. 149:114-122.

Noel, K. D., Sanchez, A., Fernandez, L., Leemans, J., and Cevallos, M. A. 1984 Rhizobium phaseoli symbiotic mutants with transposon Tn5 insertions. J. Bacteriol. 158:148-155.

Ouchane, S., Picaud, M., Therizols, P., Reiss-Husson, F., and Astier, C. 2007. Global regulation of photosynthesis and respiration by FnrL: the first two targets in the tetrapyrrole pathway. J. Biol. Chem. 282:76907699

Patschkowski, T., Schlüter, A., and Priefer, U. B. 1996. Rhizobium leguminosarum bv. viciae contains a second fnr/fix $K$-like gene and an unusual fixL homologue. Mol. Microbiol. 21:267-280.

Rey, F. E., and Harwood, C. S. 2010. FixK, a global regulator of microaerobic growth, controls photosynthesis in Rhodopseudomonas palus tris. Mol. Microbiol. 75:1007-1020.

Sambrook, J., Fritsch, E. F., and Maniatis, T. 1989. Molecular Cloning: A Laboratory Manual, 2nd ed. Cold Spring Harbor Laboratory Press, Cold Spring Harbor, NY, U.S.A.

Schäfer, A., Tauch, A., Jäger, W., Kalinowski, J., Thierbach, G., and Pühler, A. 1994. Small mobilizable multi-purpose cloning vectors derived from the Escherichia coli plasmids pK18 and pK19: selection of defined deletions in the chromosome of Corynebacterium glutamicum. Gene 145:69-73.

Schlüter, A., Patschkowski, T., Quandt, J., Selinger, L. B., Weidner, S., Krämer, M., Zhou, L., Hynes, M. F., and Priefer, U. B. 1997. Functional and regulatory analysis of the two copies of the fixNOQP operon of Rhizobium leguminosarum strain VF39. Mol. Plant-Microbe Interact. 10:605-616.

Sigrist, C. J., Cerutti, L., de Castro, E., Langendijk-Genevaux, P. S., Bulliard, V., Bairoch, A., and Hulo, N. 2010. PROSITE, a protein domain database for functional characterization and annotation. Nucleic Acids Res. 38:D161-166.

Yoshida, T., Qin, L., Egger, L. A., and Inouye, M. 2006. Transcription regulation of ompF and ompC by a single transcription factor, OmpR. J. Biol. Chem. 281:17114-17123.

Young, J. P., Crossman, L. C., Johnston, A. W., Thomson, N. R., Ghazoui, Z. F., Hull, K. H., Wexler, M., Curson, A. R., Todd, J. D., Poole, P. S., Mauchline T. H., East, A. K., Quail, M. A., Churcher, C., Arrowsmith, C., Cherevach, I., Chillingworth, T., Clarke, K., Cronin, A., Davis, P., Fraser, A., Hance, Z., Hauser, H., Jagels, K., Moule, S., Mungall, K. Norbertczak, H., Rabbinowitsch, E., Sanders, M., Simmonds, M., Whitehead, S., and Parkhill, J. 2006. The genome of Rhizobium leguminosarum has recognizable core and accessory components. Genome Biol. 7:R34.

\section{AUTHOR-RECOMMENDED INTERNET RESOURCES}

Basic NCBI/BLAST database: blast.ncbi.nlm.nih.gov/Blast.cgi

ClustalW2, Multiple Sequence Alignment database: www.ebi.ac.uk/Tools/msa/clustalw2

European Molecular Biology Laboratory SMART database: smart.embl-heidelberg.de/

ExPASy Prosite database: prosite.expasy.org/

Kazusa DNA Research Institute Rhizobase database: http://genome.kazusa.or.jp/rhizobase/Etli

MEME Multiple Em for motif elicitation database: meme.sdsc.edu/meme/cgi-bin/meme.cgi

Prokaryotic 2-Component Systems (P2CS): www.p2cs.org

Protein-Domain Sequence Analyzers database: www.ebi.ac.uk/interpro

SUPERFAMILY database: supfam.cs.bris.ac.uk/SUPERFAMILY

Wellcome Trust Sanger Institute Pfam database: pfam.sanger.ac.uk 